\title{
Skin to skin contact for very low birthweight infants and their mothers
}

\author{
A WHITELAW, ${ }^{*}$ G HEISTERKAMP, ${ }^{*} \mathrm{~K}$ SLEATH, ${ }^{*}$ D ACOLET,${ }^{*}$ AND M RICHARDS $\dagger$ \\ ${ }^{*}$ Department of Paediatrics and Neonatal Medicine, Hammersmith Hospital, London and + Child Care and \\ Development Group, University of Cambridge, Cambridge
}

SUMMARY Separation between mothers and very low birthweight infants is often prolonged with subsequent psychological distress, behaviour problems, and lactation failure. Babies as small as $700 \mathrm{~g}$, who no longer require oxygen, can be safely and enjoyably held naked, except for a nappy, between the mother's breasts for up to four hours a day. We have carried out a randomised trial among babies less than $1500 \mathrm{~g}$. Seventy one infants were randomised. In 35 , the mother was helped to hold her baby in skin to skin contact and encouraged to do so whenever she visited the baby. In 36, the mother was encouraged to handle her baby but without skin to skin contact. Mothers using skin to skin contact lactated for four weeks longer on average than the control group. At 6 months of age the infants who had skin to skin contact cried significantly less than the control group. Skin to skin contact can safely and enjoyably be offered to very low birthweight infants especially in developing countries where the mother's lactation is vital.

The decrease in perinatal mortality over the last $\mathbf{4 0}$ years has been accompanied by the rigorous precautions against sepsis that were thought to justify separation of mother and baby, and this has been particularly true with very low birthweight infants $(<1500 \mathrm{~g})$. Such infants may stay in a special care baby unit for three months and visiting by parents may be limited by the distance from home to hospital. A Perspex incubator forms an additional barrier between parent and baby for weeks or months. There has been much concern about the psychological consequences of prolonged separation on small babies and their mothers.

The value of early skin to skin contact in improving communication between mothers and full term infants has been highlighted by Klaus et al. ${ }^{1}$ Since their pioneering work many other studies have been carried out to evaluate the effects of early separation or, conversely, extra contact, on mother and baby. ${ }^{2-8}$ While results have been somewhat variable, reviews have concluded that separation does have some deleterious effects on the relationship, the frequency and duration of lactation, and the mother's feelings of confidence in being able to cope with her baby at home. Such effects have been shortlived, however, and within a few weeks or months of reunion after separation are no longer seen.
Skin to skin contact for very low birthweight infants was pioneered in Bogota, Colombia by Rey and.Martinez. ${ }^{9}$ Because of a high rate of death and abandonment in their large maternity hospital each preterm mother was encouraged to nurse her baby, however small, next to her skin between her breasts. The infants were in the head up position to minimise the risk of aspiration. This has come to be known as 'kangeroo baby care' and has received considerable interest among neonatal nurses. ${ }^{10}$ No randomised trials have been published on this type of close physical handling for such small and fragile infants.

Pilot studies showed us that stable infants as small as $700 \mathrm{~g}$ could be safely held skin to skin and that mother and baby seemed to enjoy the experience. If flexion of the infant is avoided, oxygenation is well maintained. A hat is advised for the smallest infants to maintain temperature. Mothers have made comments such as 'Now I feel he's getting to know me' and 'I feel like a mummy now'. Encouraged by these positive results we planned an objective evaluation.

This paper reports the results of a randomised controlled trial of skin to skin contact for very low birthweight infants and their mothers to test the following hypotheses: (1) early skin to skin contact improves the mother's confidence and positive feelings towards her baby when she takes the baby home and when the baby is 6 months old; (2) early 
skin to skin contact influences the infant's behaviour at 6 months of age; and (3) early skin to skin contact prolongs lactation.

\section{Patients and methods}

All infants in the Hammersmith Hospital neonatal unit were eligible for the study if they weighed less than $1500 \mathrm{~g}$, had stable breathing with no oxygen requirement, and if at least one parent spoke fluent English. Stable infants were not excluded if they had congenital abnormalities such as hydronephrosis or scoliosis, nor if they had intracranial lesions such as periventricular leukomalacia or ventricular dilatation.

Infants meeting these requirements were identified by the nursing staff and entered into the trial. Each infant was randomised by a nurse opening sequentially a numbered, sealed, opaque envelope, which contained a sheet of paper allocating the infant to either (a) skin to skin contact or (b) normal handling. The envelopes had been prepared by medical staff, AW or GH, in balanced blocks of six.

\section{MANAGEMENT OF TRIAL GROUPS}

If the infant was randomised to skin to skin contact, one of the nurses presented the idea of skin to skin contact to the mother, showing pictures if necessary. It was explained that it was a safe and enjoyable way to hold and get to know her baby but without any proven lasting benefit. Initially the mother was helped to position her baby inside her dress and between the breasts (figure) with a cardiac or respiration monitor attached. After the first two occasions, the mother was encouraged to hold her baby in this position whenever she came to visit and to continue this close contact for the rest of the time her baby was in Hammersmith Hospital and later.

If the infant was randomised to normal contact, the mother and baby had the same amount of support and help as the skin to skin contact group. The mother was encouraged to visit as much as she liked and helped to take her baby out of the incubator for a cuddle. However, baby and mother remained clothed. Care was taken that the normal contact group would have no less attention from the nursing staff for talking, listening, questions and answers.

In cases of twin pregnancy the first twin who was ready to enter the trial was randomised as described above. If the other twin became eligible for the trial, the new trial entrant was allocated the same handling as the first twin as it was felt that it was unacceptable to ask a mother to handle one twin differently from the other one. If an infant in the trial became unwell, the trial was discontinued until the infant was stable again. This applied equally to both handling groups.

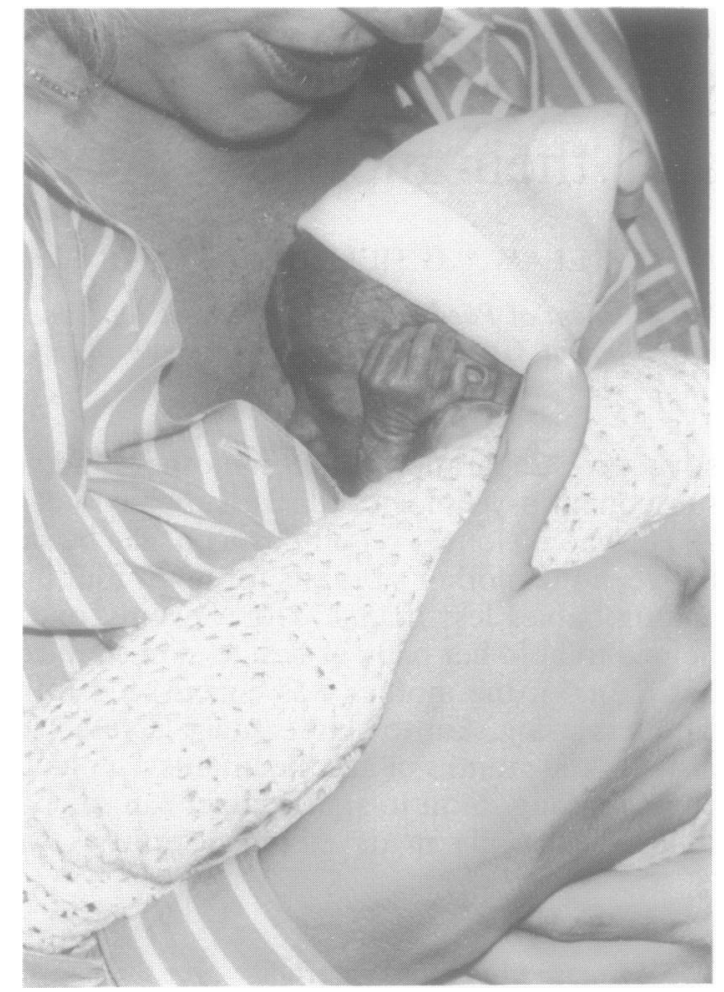

Figure An infant girl, born at 27 weeks' gestation and weighing $880 \mathrm{~g}$, held in skin to skin contact by her mother at 2 weeks postnatal age.

Each time the mother visited, a record was kept of the duration of the visit, normal (that is, clothed) contact, and skin to skin contact.

\section{MEASUREMENT OF OUTCOME}

When the infant was about to be discharged from Hammersmith Hospital, either to go home or back to the referring hospital, the mother was given a questionnaire. The mothers were asked to rate themselves on a six point scale. The questions covered confidence looking after the baby, knowing the baby well, feeling optimistic about the baby, being depressed, feeling detached from the baby, and feeling supported in looking after the baby. Each mother was also asked if she had thought at any time that her baby was going to die.

All infants in the trial were seen in the routine neonatal follow up clinic at 6 months of age (uncorrected for prematurity). Again, mothers were asked to answer a questionnaire on feelings about the baby on a six point scale. The questions concerned: feeling detached from the baby, know- 
ing what the baby wants, whether the baby expects to be carried around, whether the baby is contented, feeling annoyed with the baby, whether the baby is easier to look after than she had thought in hospital, support in looking after the baby, being worried that something will still happen to the baby, and feeling that the baby's behaviour has completely caught up with full term babies. Each mother was also asked when she thought her baby was really hers.

Duration of lactation was determined by history from the mother at discharge, or at six months. If the mother was still lactating at six months, the duration of lactation was determined at the nine or 12 month routine visit.

At six months the parents were asked to keep a 48 hour diary of the infant's behaviour. Duration and frequency of sleeping, feeding, being held, and playing were recorded by the parents to the nearest 15 minutes. Playing was defined as the infant being awake and taking notice of the surroundings but without crying, being held, or fed. Duration and frequency of crying were recorded to the nearest five minutes. When the parents were unable to come to the neonatal follow up clinic, the questionnaires and diaries were sent and returned by post. In 12 cases, a home visit by $\mathrm{AW}$ was necessary to complete the information. Follow up data were obtained for all surviving infants.

Before the randomised trial started, we piloted the questionnaires and the diary with mothers of very low birthweight infants. Minor modifications to the original layout were made as a result of the pilot study.

\section{SAMPLE SIZE}

We previously had found $32-33 \%$ of mothers of very low birthweight infants were still lactating at six weeks. To have an $80 \%$ chance of detecting a doubling of this proportion to $65 \%$ (at $\mathrm{p}<0.05$ ) would require 36 subjects in each group. We aimed to recruit 80 infants. Over an 18 month period, August 1985-February 1987, 71 infants were entered in the study. This represents about half of all the very low birthweight infants admitted to the Hammersmith Hospital neonatal unit during that period. The reasons for the remaining infants not being entered were: prolonged respiratory problems, early transfer back to the referring hospital, early neonatal death, and neither parent speaking fluent English. Because of the number of multiple pregnancies and four deaths, 71 infants recruited to the study gave a total of 63 mothers available to give a lactation history.

Statistical analysis was carried out on a Perkin Elmer 3220 computer using Minitab statistical pack- ages. Where data had a Gaussian distribution and similar variances, means were compared using the unpaired Student's $t$ test. Where data did not have a Gaussian distribution, comparisons were made using the Mann-Whitney $U$ test and $\chi^{2}$ test.

The study was approved by the Hammersmith Hospital research ethics committee.

\section{Results}

Table 1 shows the characteristics of the infants and mothers at entry to the trial. Thirty five infants were randomised to the group with skin to skin contact and 36 to group with normal handling. There was no significant difference in birth weight, gestational age, sex, Apgar scores, caesarean section rate, parity, previous neonatal death, ethnic group, social class, or lack of partner support. Thus the two groups were similar for many important factors that could affect the mother's relationship with her child in the first few weeks. The infants entered the trial on average on the 16th day (there was a range from the first to 61st day for the group with skin to skin contact and from the the first to 66th day for'the group with normal handling). The numbers of mothers originally wishing to breast feed were similar $(24(77 \%)$ in the group with skin to skin contact and $26(81 \%)$ in the group with normal contact). Those in the group with skin to skin contact remained at Hammersmith for a median $\mathbf{3 0}$ days (range 5-83 days) and those in the group with normal contact remained a median 37 days (range 5-78 days).

Table 2 shows details of the visiting. Average

Table 1 Characteristics of infants at entry to trial

\begin{tabular}{lcc}
\hline & $\begin{array}{c}\text { Group with } \\
\text { skin to skin } \\
\text { contact }\end{array}$ & $\begin{array}{l}\text { Group with } \\
\text { normal } \\
\text { handling }\end{array}$ \\
\hline No of infants & 35 & 36 \\
Male/female & $21 / 14$ & $14 / 22$ \\
Singleton & 26 & 27 \\
Mean (SD) birth weight (g) & $1152(220)$ & $1135(263)$ \\
$\quad$ Range & $560-1500$ & $630-1500$ \\
Mean (SD) gestational age (weeks) & $29 \cdot 1(2 \cdot 3)$ & $29 \cdot 5(2 \cdot 3)$ \\
$\quad$ Range & $26-35$ & $25-36$ \\
Mean Apgar score at 1 minute & $5 \cdot 6$ & $5 \cdot 5$ \\
Caesarean section & 23 & 23 \\
Primiparous & 21 & 19 \\
Previous neonatal death & 2 & 2 \\
White & 26 & 24 \\
Asian & 5 & 8 \\
Afro-Caribbean & 4 & 4 \\
Unsupported mother & 2 & 4 \\
Intention to breast feed & 24 & 26 \\
\hline
\end{tabular}


Table 2 Handling and visiting by parents in the neonatal unit during the trial

\begin{tabular}{|c|c|c|}
\hline & $\begin{array}{l}\text { Group with } \\
\text { skin to skin } \\
\text { contact } \\
(n=35)\end{array}$ & $\begin{array}{l}\text { Group with } \\
\text { normal } \\
\text { handling } \\
(n=36)\end{array}$ \\
\hline Mean (SD) visiting/day (hours) & $2 \cdot 1(0 \cdot 8)$ & $2 \cdot 2(0.9)$ \\
\hline Range & $0.7-3.9$ & $0.7-11.0$ \\
\hline $\begin{array}{l}\text { Median time left in incubator } \\
\text { while mother visited (hours) }\end{array}$ & $0 \cdot 1$ & $0 \cdot 1$ \\
\hline Range & $0-2 \cdot 0$ & $0-5 \cdot 9$ \\
\hline $\begin{array}{l}\text { Mean (SD) time touched or } \\
\text { cuddled with clothes on (hours) }\end{array}$ & $1.4(0.7)$ & $1.8(1.0)$ \\
\hline Range & $0 \cdot 2-3 \cdot 4$ & $0 \cdot 5-5 \cdot 2$ \\
\hline $\begin{array}{l}\text { Median time skin to skin } \\
\text { contact (hours) } \\
\text { Range }\end{array}$ & $\begin{array}{l}0.6 \\
0-1 \cdot 5\end{array}$ & - \\
\hline
\end{tabular}

visiting was just over two hours a day, most of it spent holding the infant clothed. Visiting time was limited by the long distance from some homes to the hospital, lack of a family car, the needs of older children at home, and by illness of the mother. The group with skin to skin contact had, on average, 36 minutes per day of skin to skin contact. Breast feeding time was not counted as skin to skin contact time. Skin to skin contact was declined by five mothers, three of them Asian and two white, but these infants remained in the group with skin to skin contact for analysis of outcome in order not to violate the randomisation. There was no difference overall between the two groups in total visiting time.

Temperature instability was not a problem during skin to skin contact. The trial had to be discontinued temporarily in six infants in each group because of apnoeas, necrotising enterocolitis, or sepsis. Two infants died in each group between entering the study and reaching 6 months of age. Two infants died of septicaemia, one had necrotising enterocolitis, and one had a sudden infant death. This underlies the high risk nature of the population we are dealing with.

When questionnaires at discharge and at six months were analysed, there was no significant difference between the two groups on any of the six point psychological scales. It is noteworthy that 48 $(67 \%)$ of the mothers overall thought that at some time their baby was going to die, and that $30(42 \%)$ did not feel that the baby was really theirs until he or she was home.

Table 3 shows the behavioural findings from the diary kept when the infants were 6 months of age. The duration of sleeping, feeding, being held, and playing were almost identical in the two groups. Median duration of crying was 25 minutes per day in
Table 3 Behavioural findings in the infants at 6 months of age

\begin{tabular}{lcc}
\hline & $\begin{array}{l}\text { Group with } \\
\text { skin to skin } \\
\text { contact } \\
(n=35)\end{array}$ & $\begin{array}{l}\text { Group with } \\
\text { normal } \\
\text { handling } \\
(n=36)\end{array}$ \\
\hline Mean (SD) hours sleeping/day* & $13 \cdot 6(2 \cdot 3)$ & $13 \cdot 4(2 \cdot 3)$ \\
Mean (SD) hours feeding/day* & $2 \cdot 5(1 \cdot 1)$ & $2 \cdot 5(0 \cdot 9)$ \\
Mean (SD) hours being held/day* & $3 \cdot 0(1 \cdot 3)$ & $3 \cdot 0(1 \cdot 4)$ \\
$\begin{array}{l}\text { Mean (SD) hours playing/day* } \\
\text { Median (range) minutes }\end{array}$ & $4 \cdot 5(1 \cdot 8)$ & $4 \cdot 5(1 \cdot 8)$ \\
crying/day $\dagger$ & $25(0-100)$ & $38(5-140)$ \\
\hline
\end{tabular}

${ }^{*}$ Gaussian distributions: no difference between means was significant at $\mathrm{p}<0.05$ by Student's $t$ test.

†Non-Gaussian distribution: Mann-Whitney U test $p=0.0422$.

the group with skin to skin contact and 38 minutes per day in the group with normal handling. This difference was significant by the Mann-Whitney $U$ test $(p=0 \cdot 0422)$.

Mean duration of lactation in the group with skin to skin contact was 9.2 weeks (median 7 weeks) whereas, in the group with normal handling, mean duration of lactation was 5.1 weeks (median 3.5 weeks). The results for the two groups are significantly different (Mann-Whitney U test, $p=0 \cdot 0167)$. Furthermore, 17 out of 31 mothers $(55 \%)$ in the group with skin to skin contact lactated for more than six weeks; this compares with only nine out of $32(28 \%)$ in the group with normal handling $\left(\chi^{2}\right.$ $\mathrm{p}<0 \cdot 02)$.

\section{Discussion}

The intervention in this randomised trial consisted of presenting and encouraging early skin to skin contact in a busy regional neonatal intensive care unit where mothers are already encouraged to visit whenever they like, siblings can visit, and there is a generally relaxed and supportive atmosphere. Introducing skin to skin contact did not significantly prolong total visiting time. Apart from the five mothers who declined, each mother and baby seemed to enjoy skin to skin contact, but we were unable to detect any psychological advantage when the baby was discharged from our hospital or at 6 months of age. In contrast to the study by Klaus $e t$ $a l,{ }^{1}$ our trial involved infants under $1500 \mathrm{~g}$ rather than full term infants and all our mothers had a lot of other forms of emotional support as well as unlimited access to see and handle their infants (which was not the case in the study of Klaus $e t$ al in Cleveland, Ohio). We conclude that early skin to skin contact does not seem to be more effective than other forms of contact and general support in giving 
mothers of very low birthweight infants longer term confidence and positive feelings.

On average the very low birthweight infants with skin to skin contact, aged 6 months, cried for 13 minutes per day less than the group with normal handling. Many parents would rate this a valuable advantage. Regular skin to skin contact may give the infant security and tranquility, which is reflected in less crying subsequently. Infants who had had early skin to skin contact did not demand to be held more than the control group at 6 months of age and were not carried around more by their mothers.

The four week increase in duration of lactation and the proportion of mothers lactating for more than six weeks is an important finding and is in agreement with De Chateau and Wiberg's trial of skin to skin contact for full term infants. ${ }^{2}$ Presumably an average of 36 minutes a day of close physical contact between the baby and both breasts is enough to increase the secretion of prolactin. Even in developed countries mother's milk is widely regarded as the most suitable food for very low birthweight infants. ${ }^{11}$ It can be given raw to preserve its protective substances and supplements of sodium and minerals can be given as necessary. If the mother is able to maintain her lactation for six weeks then there is a good chance that she will be able to breast feed the baby at home. In developing countries, mother's milk is much more important, literally life saving, because of the relatively high cost of milk formulas and the risk of diarrhoea from contaminated milk. ${ }^{12}$ In the Third World, the use of 'kangaroo baby care' may be an important way of maintaining the mother's precious lactation.

We are grateful to the nursing staff of the Hammersmith Hospital neonatal unit and to the parents and babies studied. Dr Acolet was supported by a grant from Foundation pour la Recherche Medicale, Paris.

\section{References}

${ }^{1}$ Klaus MH, Jerauld R, Kreger NC, McAlpine W, Steffa M, Kennel JH. Maternal attachment. The importance of the first post-partum days. $N$ Engl J Med 1972;286:460-3.

2 De Chateau P, Wiberg B. Long term effect on mother-infant behaviour of extra contact during the first hour post-partum. Acta Paediatr Scand 1977;66:137-51.

${ }^{3}$ Goldberg S. Parent-infant bonding: another look. Child Dev 1983;54:1355-82.

${ }^{4}$ Lamb ME. Early mother-neonate contact and mother-child relationship. J Child Psychol Psychiatry 1983;24:487-94.

${ }^{5}$ Ross GS. Parental responses to infants in intensive care. The separation issue re-evaluated. Clin Perinatol 1980;7:47-60.

6 Richards MPM. Parent-child relationships: some general considerations. In: Davis JA, Richards MPM, Roberton NRC, eds. Parent-baby attachment in premature infants. London: Croom Helm, 1983.

7 Sluckin W, Herbert NJ, Sluckin A. Maternal bonding. Oxford: Blackwell, 1983.

8 Trause MA. Extra post-partum contacts: an assessment of intervention and its effects. In: Smeriglio VL, ed. Newborns and parents. Hillsdale, NJ: Erlbaum, 1981.

9 Whitelaw A, Sleath K. Myth of the marsupial mother: home care of very low birth weight babies in Bogota, Colombia. Lancet $1985 ; \mathbf{i}: 1206-8$.

10 Anderson GC, Marks EA, Wahlberg V. Kangaroo care for premature infants. Am J Nurs 1986:807-9.

11 Wharton B, ed. Nutrition and feeding of preterm infants. Oxford: Blackwells, 1987.

12 Victoria C, Smith P, Vaughan J, et al. Evidence for protection by breast feeding against infant deaths from infectious diseases in Brazil. Lancet 1987;ii:319-21.

Correspondence to Dr A Whitelaw, Department of Paediatrics and Neonatal Medicine, Hammersmith Hospital, DuCane Road, London W12 0HS.

Accepted 16 May 1988 